Science 4 April 2014:

Vol. 344 no. 6179 pp. $46-48$

DOI:10.1126/science.1246251

Perspective

\title{
To Fence or Not to Fence
}

Rosie Woodroffe, Simon Hedges, Sarah M. Durant I 2 Comments

Fences must be used with care in biodiversity conservation to avoid unintended consequences.

\section{Comments}

\section{Joao Pedro Barreiros}

R. Woodroffe et al. Perspective in Ecology "To Fence or Not to Fence" (4 April, 344: 46-48) state a definitely important conclusion when emphasizing two main aspects: a) fence removal may become an important form of climate change preparedness and b) fencing of wildlife should become an action of last resort. This same problem does apply to a vast amount of potentially dramatic and understudied effects when projecting these same concerns to Marine Protected Areas (MPAs). In fact, those worries are expressed by (1) when explaining connectivity between MPAs and the fact that, in spite of no fencing being viable underwater, corridors in between adjacent MPAs may well work as fences since fishing pressure, either professional or recreational, do create "fences" that inhibit the beneficial effect of biomass exports from MPAs to outside areas as explained by modelling these areas in different sizes either vast (2) or "giant" ones (3). To this, one has to add the objectives that led to the implementation of MPAs (4) which do vary and show significant differences, both positive and negative, while many did indeed surprise their own proposed designers and outcome objectives. While limiting MPAs by charts may be of low practical use due to numerous factors (e.g. fishermen's inability to read charts and/or to operate a GPS), the issue still connects to the problem of "fencing". Of course, fences will not be a reliable (or even technically possible) way of delimiting MPAs but the above mentioned corridors in between them, allowing a number of fishing methods, do act ecologically as "fences". This understudied and often discarded approach may well be the key to a proper design of the Worlds' MPAs while assuring the indispensable connectivity between them.

References 1. M.N. Dawson et al., Science 313, 43-44 (2006). 2. J.N. Sanchirico et al., Science 301, 47-48 (2003). 3. D.M. Kaplan et al., Science 340, 810-811 (2013). 4. C.M. Roberts et al., Science 294, 1920-1923 (2001). 


\section{Science Online Home | News | Science}

Journals | Careers | Multimedia | Collections | Help | Site

Map | RSS

Subscribe | Feedback | Privacy Policy | About Us | Advertise With

Us | Contact Us

(C) 2010 American Association for the Advancement of Science. All

Rights Reserved.

AAAS is a partner of HINARI, AGORA, OARE, Patientlinform. 